\title{
Automation on the move
}

\author{
The insatiable demands of the pharmaceutical industry are helping to drive labs into the \\ automated world of industrial production lines. Tim Chapman joins the quest for speed.
}

$\longrightarrow$ ince clinical laboratories began using robotics in the early 1980 s to manage their thousands of samples a day, automated systems have become commonplace. By rapidly and tirelessly performing processes that would have been done manually — from sample storage and retrieval to running customized assays modern automated systems can greatly increase the throughput of a laboratory, free up researchers from repetitive tasks, and monitor and manage the raw data produced.

Automation is now increasingly common in agricultural, food and environmental analytical labs, but it is the demands of the pharmaceutical industry for high-throughput screening of potential drug candidates (see Nature 418, 453-459; 2002) that is most influential in driving the technology forward. Researchers now have whole genomes of potential drug targets at their disposal for testing against an array of drug candidates, and high-throughput automation is rapidly becoming a necessity.

"Automation is used in a broad range of approaches in pharmaceuticals, from early combinatorial creation to high-throughput screening," says Robin Felder, director of the Medical Automation Research Center at the University of Virginia in Charlottesville and president of the Association for Laboratory Automation. "The skills of creating a new drug start with synthesizing novel molecules - it used to be one scientist, one molecule, one week. Now, one molecular chemist can turn out 100 good compounds a week."

\section{The fast track}

Many pharmaceutical and biotechnology companies would be unable to function without high levels of automation. A typical end-user is Cyprotex, based in Macclesfield, UK, which aims to improve the efficiency of drug discovery by using industrial-scale testing and proprietary software to predict how drug candidates will act in the body.

The assays that could rule out an otherwise promising drug candidate are for absorption, distribution, metabolism and excretion. To avoid a bottleneck in the drug-discovery process, these assays have to be carried out as rapidly as the drug candidates are identified. "We have automated the assays, which are currently

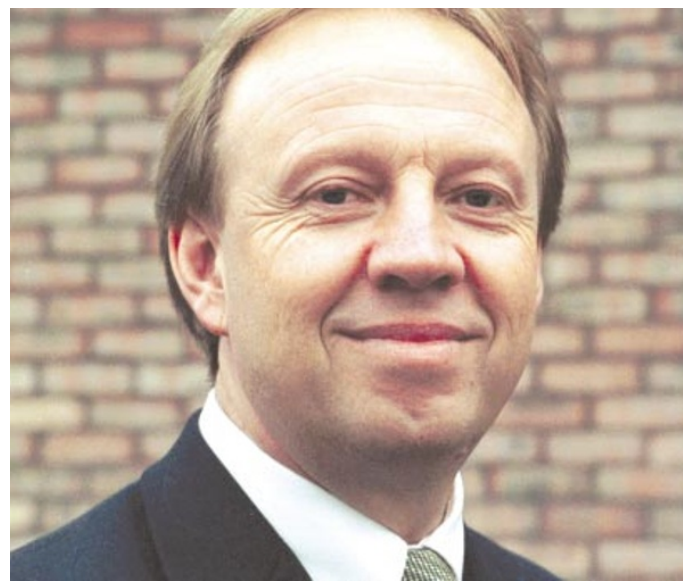

David Leahy: screening can benefit greatly from automation.

running at 100-200 compounds a week in major pharmaceutical companies, to where we can run 2,000-5,000 compounds a week," says David Leahy, founder and chief scientific officer at Cyprotex. "We think there's a potential for running, if we pick one assay, say 750,000 compounds a year."

\section{A STRUCTURED APPROACH}

Mapping the detailed atomic structure of a target protein is vital for identifying drug candidates that can bind with it. Elucidating protein structure by crystallography has for years been a laborious operation carried out by specialists, but automation is now beginning to bring the process up to the speeds demanded by high-throughput systems.

Automated crystallography systems for the general research market have been available for some time from specialists such as Tecan in Männedorf, Switzerland, and BSI Proteomics of Gaithersburg, Maryland. But dedicated drug-discovery companies are now forcing the pace, with the vast majority of commercial protein crystallography done by just two companies, Syrrx and Structural GenomiX, both based in San Diego.

Syrrx wants to automate every stage of protein-structure determination. It deploys a family of whimsically named robots, beginning with a highthroughput robot called Sonic Hedgehog that can purify about 100 proteins in five hours. The proteins are then crystallized by Agincourt, a robot named for English king Henry V's application of superior technology to overcome extreme odds. Agincourt crystallizes proteins from droplets of just 50 nanolitres, about a fortieth of the volume of previous systems. "Everything is miniaturized," says business development director Ned David. "These droplets enable our whole approach to work in a way that is economically feasible. You can't do nano-drops without automation because you can't set your pipette for 50 nanolitres."

To match the demands of Agincourt, Syrrx has two imaging-station robots called Fort Knox and Fort Bliss, each capable of scanning over a

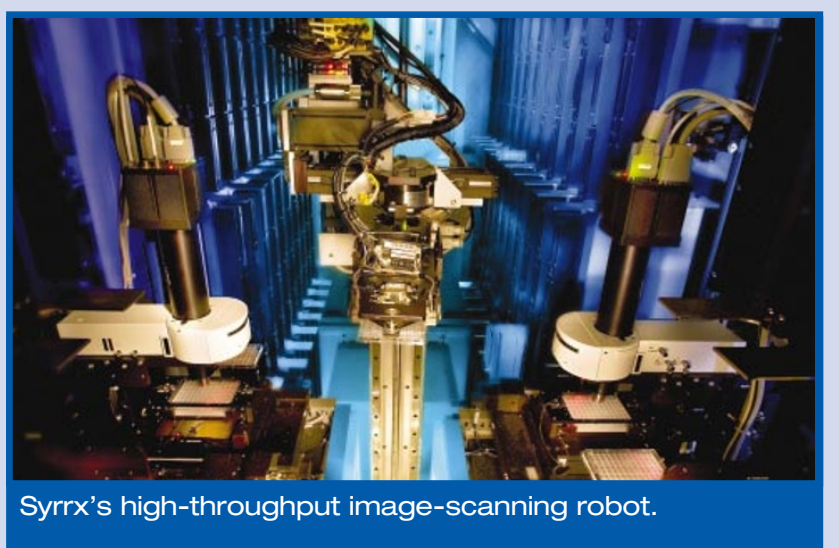

million images a day for suitable protein crystals. These are then taken to Syrrx's dedicated synchrotron beamline at the Advanced Light Source in Berkeley for X-ray diffraction. Data are collected by another robot called Robohutch, which David says can increase throughput at this stage tenfold.

There is still scope for further automation, particularly in the first stages of protein production. Syrrx is investigating how more mammalian proteins can be made in eukaryotic cell systems, rather than from genes cloned in bacteria. T.c. 
But this level of automation brought technical challenges. Specialist consultancy The Automation Partnership (TAP) in Royston, UK, worked with Cyprotex to adapt TAP's proprietary BasePlate liquidhandling robots to Cyprotex's particular requirements. Cyprotex also automated many of its analytical instruments to run standardized tests on compounds coming through the high-throughput process.

Cyprotex is now in a position to extend its automated systems to carry out a wider range of important assays. "We think automation gives us a screening capacity that is at least an order of magnitude higher than a typical pharmaceutical company, at a unit cost that is possibly 20 -fold less," says Leahy.

\section{Small is beautiful}

Benchtop automated liquid handling and sample-dispensing systems are now routine in most life-science laboratories. Such systems will become even more ubiquitous with the introduction of a new wave of lower-cost modular devices with much the same functionality as the systems used by big-pharma labs. "The pharma labs have the deepest pockets, but a new generation of automated systems is becoming available that's more the 'Volkswagen' of the market," says Felder.

Xiril, a young Swiss company founded in Hombrechtikon by a team of specialists in robotic liquid-handling, has a range of pipetting robots that costs about $20-30 \%$ less than comparable systems. The Xiril robots are primarily aimed at low-tomedium-throughput operations, particularly for processes that have not yet been automated.

"We believe we can fill a market for liquid-handling processes that are often done manually, even in labs that are highly automated," says the firm's chief executive Heinz Abplanalp. Xiril has also developed automated systems for magnetic-bead separation.

At the more expensive end of the liquid-handling market, the trend is towards increasing miniaturization. Given the high cost of many reagents used in biomedical experimentation, considerable savings can be made simply by using less. Allegro Technologies, a Dublin-based company spun out of Trinity College, has the proprietary technology to dispense droplets up to 1,000 times smaller than those from standard equipment. The company claims that this could reduce the cost of some experiments sixfold.

Allegro's latest range of pipetting systems uses electromagnetically controlled valves to deliver droplets of 20 microlitres down to 20 nanolitres in volume. The firm has licensed its technology to US instrument manufacturers Gilson of Middleton, Wisconsin, and Beckman Coulter in Fullerton, California, and has also put the pipettes into its own robotic system.

Being able to manipulate tiny volumes of liquid means that entire lab processes can be miniaturized. Using technology developed for the semiconductor industry, chips a few centimetres square can be etched with microscopic channels in which routine preparation procedures and assays can be carried out.

Caliper Technologies in Mountain View, California, was the first to market these 'lab-on-a-chip' devices. Its 250 HTS system can use a range of chips for enzymatic or cell-based drug screens; some assays require fewer than 500 cells.

Caliper's chips use small electrical currents or vacuum-based pressure techniques to move fluids through the channels. Gyros in Uppsala, Sweden, is developing a range of microlabs for processes such as protein purification in the form of compact discs. A robotic workstation loads the CD with reagents and spins it at precisely controlled speeds, driving the fluids through the channels by centrifugal acceleration. Gyros's first product, the Gyrolab MALDI, can prepare up to 96 protein samples simultaneously for mass spectrometry. Tecan has also launched a similar product — its LabCD devices for pharmacological assays.

\section{Action stations}

Workstations, stand-alone automated solutions for specific lab tasks such as DNA sequencing or immunoassay tests, continue to be dominated by established

\section{CULTURAL REVOLUTION}

Cell culture, either for the cells themselves or their products, is traditionally a manual process that demands hours of repetitive, painstaking work to ensure absolute sterility under exacting conditions. Growing a mammalian cell culture for use in a screen for drug activity or toxicity typically takes 48 hours, so almost half the working week is gone before screening can begin.

But with automated cell-culture systems, screening could start on Monday morning on cultures left to grow over the weekend. Robotic systems can also provide a consistency of procedure and sterility that the best technicians cannot match, ensuring less variability between batches.

The Automation Partnership (TAP) in Royston, UK, introduced its pioneering Cellmate workstation in the late 1980s for the relatively large-scale production of therapeutic reagents and vaccines. This has now been joined by a new system, SelecT, developed in association with a consortium of six major drug companies, including GlaxoSmithKline and Pfizer.

SelecT is aimed at labs that need an automated system for processing up to 168 different mammalian cell lines simultaneously, making it ideal for multiple batches. The system can automatically maintain, expand, process and harvest multiple cell lines, assess their viability, and distribute them onto a maximum of 300 microtitre plates. "Cellmate is not so good at dealing with just-in-time production of ready-to-go microplates," says Mark Beggs, head of consulting at TPA. "SelecT can handle all the capabilities you need to take cells through to incubation to microplates."

Other commercial systems provide an even greater level of integration. Cytogration, a subsidiary of Brandel in Rockville, Maryland, has developed a robotic system that performs both cell culture and screening assays for drug candidates. The standard system handles up to 504 plates, and automates cell production, preparation of membrane-bound cells and in vitro screening.

An automated system has primarily to maintain a stable environment and reduce the risk of contamination, says Sean Sales, applications consultant at RTS Life Sciences in Manchester, UK. The system also needs to be dynamic in terms of knowing everything, so that the robot itself isn't slowing things down, he says. RTS has developed a flexible cell-culture system called acCellerator which has a very simple liquid-handling method and three parallel robot arms to process the flasks and plates in parallel. "It has a number of peristaltic pump units that allow a very short liquid line for reagents, so you don't have a lot of tubing to change over between cell lines," says Sales.

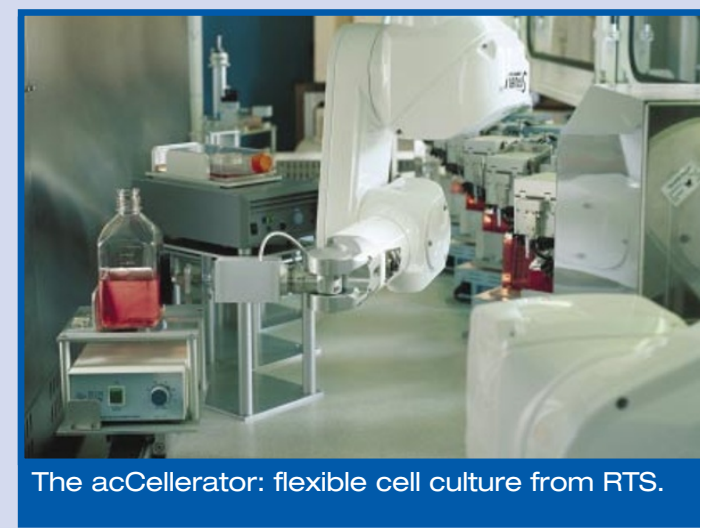


names. The Biomek FX liquid-handling workstation from Beckman Coulter, used extensively in DNA preparation, is a sophisticated descendant of the Biomek 1000 system introduced in 1986 . The latest model can simultaneously automate three liquid handlers on the same unit.

The popular BioRobot series of automated workstations made by QIAGEN Instruments near Zurich, Switzerland, offers a range of dedicated platforms for highthroughput genomics. The most recent entrant, the BioRobot Protein LS system, integrates all the software and hardware needed to purify and prepare proteins for study using X-ray crystallography or nuclear magnetic resonance.

Zymark, based in Hopkinton, Massachusetts, continues to develop its Staccato range of liquid-handling workstations, based around the Sciclone liquid-handling robots. The Sciclone ALH3000, launched in autumn last year, can dispense into microplates of up to 1,536 wells, with a new head design that can be rapidly switched to pipette volumes of 1 millilitre down to 100 nanolitres.

"The general trend we are seeing is to integrate a greater number of functions into a single system that can carry out a related set of applications," says Mark Roskey, vice-president of marketing at Zymark.

Advances in detection technology are also boosting the capabilities of workstations. The plate::screen workstation

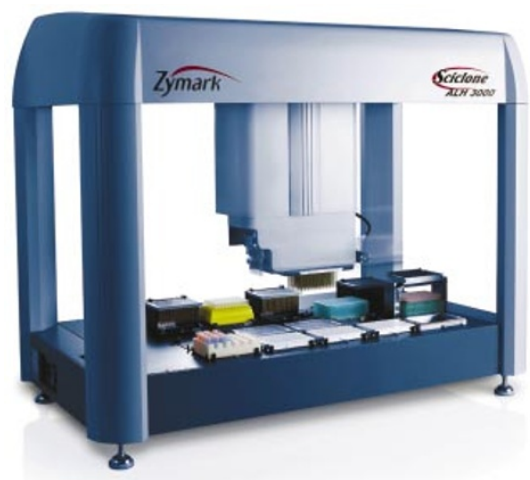

\section{Zymark's latest Sciclone robot brings} added flexibility to liquid handling.

for microplate processing, launched last year by Carl Zeiss of Jena, Germany, incorporates the first commercially available multimode detection system to deliver highperformance data from microplates of up to 1,536 wells. The plate::vision detector reads 96 wells in parallel with three detection modes - absorbance, fluorescence and luminescence - allowing it to analyse a 1,536-well plate in 20 seconds.

\section{All together now}

In theory, high-throughput systems should be able to screen about 100,000 compounds a day against a single disease target. But such rates can rarely be maintained consistently because of problems and delays in transferring sample plates between individual tests and preparation processes. Drug companies typically screen about 25,000 compounds a week, says Mark Beggs, head of consulting at TAP. "What that's really telling you is that coordination of subordinate processes is less than optimal.'

Integrating the various stages of automation into a laboratory can mean that researchers have to adapt to a more industrial production-line environment. "The hard changes are organizational ones, and getting people away from a researchdriven culture to a production-driven culture," says Beggs. That requires attention to bottlenecks and the time taken to transfer plates between individual stages rather than just buying a faster robot, he says. "While some companies get faster by buying the latest thing from the box, more are increasingly becoming faster through process-time reduction."

In addition, commercial highthroughput screening demands extreme flexibility and robustness - a typical screening department in a major drug company will run 20-50 new assays a year against a library of up to 1 million compounds, with each assay involving up to 100 separate activities. Downtime for reconfiguration or replacement of an automated system can significantly reduce effective throughput. Research by automated solutions provider RTS Life Sciences of Manchester, UK, suggests that

\section{SAMPLED DELIGHTS}

One of the remaining challenges for high-throughput automation comes at the beginning of many processes - the storage and retrieval of samples. Keeping track of samples can be a major headache for many labs, with researchers often spending hours locating a single specimen.

A pioneer in this field is Biophile, based in Charlottesville, Virginia. Biophile has developed a fully automated storage and retrieval system, which operates at temperatures down to the $-80^{\circ} \mathrm{C}$ required for storing materials such as RNA and tissue samples. The unit has built-in robotics that pick from a rotating vertical stack of almost 1,000 microplates or racks of cryogenic vials.

This month, the company is launching its individual vial retriever, which can automatically re-rack, scan, weigh and sort vials. The retriever will have a capacity of over 22,000 cryovials, which it can select and deliver at an average rate of 200 per hour. "This could double the productivity and rate of working with samples," says Sean Graves, the company's vice-president for technology.

The storage system is designed to be compatible with a wide range of equipment, and the vial retriever is designed to work with LIMS and to take samples directly to workstations by a conveyor system. As with LIMS, the demand for automated storage systems will be increased by regulatory pressure for drug companies and research labs to maintain the security of medical specimens for long periods of time.

"Automated storage and retrieval systems is the clear application that's going to surprise everyone," says Robin Felder, president of the Association for Laboratory Automation. "It's going to surprise everyone because you're going to have to have this."

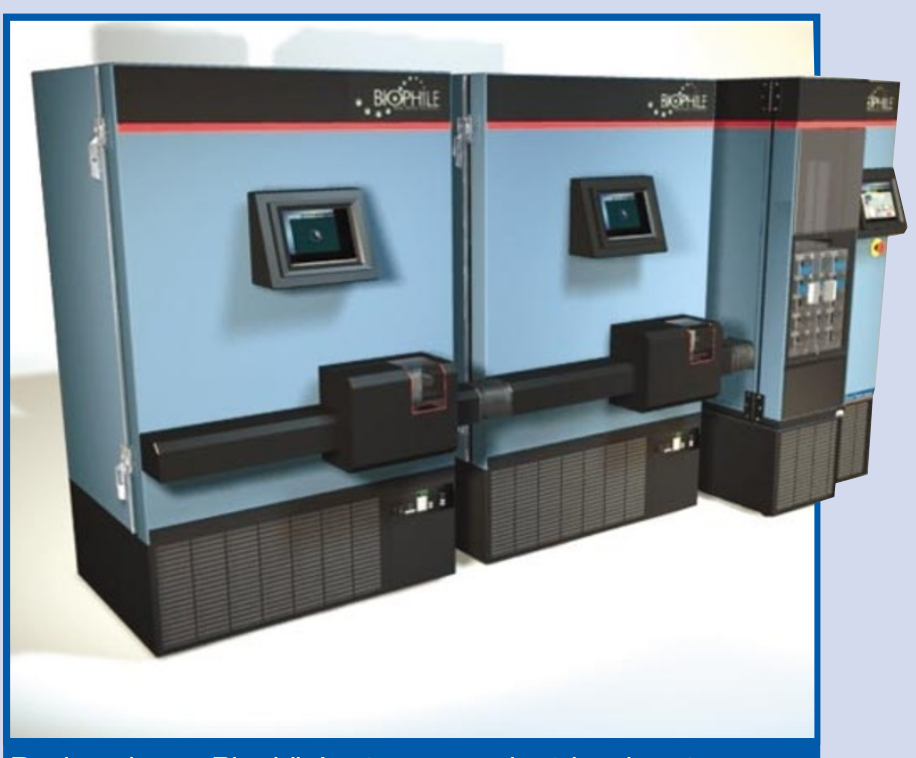

Boxing clever: Biophile's storage and retrieval system. 
each high-throughput screening system typically has an instrument replaced every six months because of the continual advances in instrument technology, meaning that research is frequently put on hold while the system is upgraded.

Some parts of common high-throughput screening processes have proved harder to automate than relatively simple tasks such as sample dispensing. Protein crystallography and cell culture, for example, are now opening up to full automation (see 'A structured approach', page 661 and 'Cultural revolution', page 663), whereas others, such as high-throughput mass spectrometry, remain more intractable.

Some automation providers have improved the overall throughput of a complex process by creating a series of specialized platforms that can be fully integrated to track each sample and its accompanying data through the whole process. The BioCube platforms from Protedyne in Windsor, Connecticut, are designed to address the worst bottlenecks in biotech processes. The range so far tackles liquid handling, the polymerase chain reaction, colony and plaque picking, and gel electrophoresis. The most recent addition is the SNPCube, which has a daily identification rate of up to 90,000 singlenucleotide polymorphisms - the single base differences between individuals that are sometimes linked to disease.

"The BioCube can become a complete system where data and processes are traced from the second the first plate enters the system," says European product manager Volker Knack. Eight- to tenfold increases in throughput can be achieved across the entire drug-discovery process, Knack says.

\section{Organization}

The development of automated systems in the life-science laboratory has gone handin-hand with the information-management systems needed to handle the masses of data generated. But the conventional laboratory information management systems (LIMS) developed for analytical chemistry operations are generally too inflexible for bioscience work. As well as producing a greater volume and complexity of data, bioscience researchers usually need a wider view of the relationships between data sets.

This has led to a new generation of systems being designed by a range of firms from specialized software developers such as L.I.M.S. in Hollywood, Florida, with its StarLIMS package, to IBM Life Sciences with its DiscoveryLink data-integration system.

"LIMS holds it all together," says Felder. "The LIMS area is actually evolving itself it used to be gathering data and organizing it, but it's now getting to be integrated so

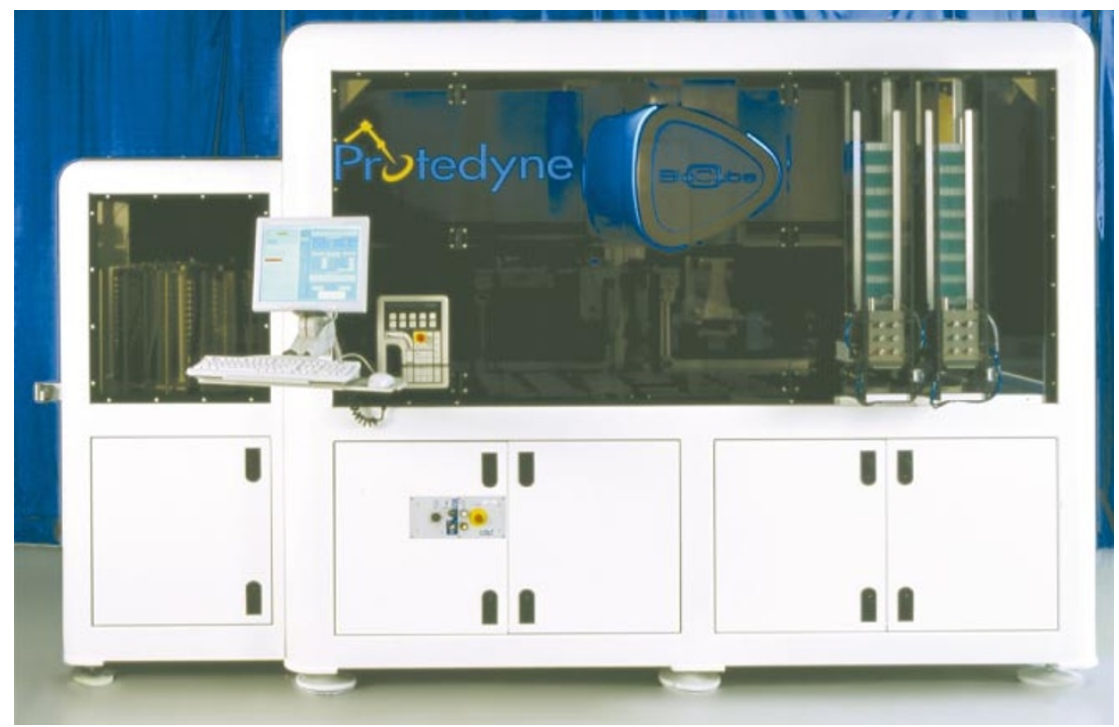

Protedyne's BioCubes are aimed at easing bottlenecks in lab processes.

you can be at a single workstation and view what everyone is doing."

The newest entrant to this market is the Scierra Laboratory Workflow System range from Amersham Biosciences in Piscataway, New Jersey, which is aimed at high-throughput genomics research. As laboratories scale up, the amount of data they generate increases hugely, as does the challenge of extracting useful information from the data (see Nature 419, 751-757; 2002). Scierra is designed to mirror the natural workflow of a lab, linking manual and automated processes into one integrated management system.

The Scierra system interfaces closely with the software on dedicated automated equipment such as Amersham's

MegaBACE DNA sequencer, so that additional equipment can be integrated into the system. But equipment from other suppliers may not be compatible, either because individual instruments don't provide a suitable interface or because their controlling software cannot be programmed to talk to other systems.

"At this stage of development, most LIMS cannot fully drive a whole lab process, so there's still an amount of human involvement," says John Schneider, vice-president for marketing at Amersham's informatics division.

Integrating LIMS with a range of automated platforms can demand considerable programming skills. The Nautilus system developed by Thermo LabSystems, based in Altrincham, UK, is designed to simplify the process by converting data from instruments and other applications into a common XML-based file format. "This means it can handle the various robotics in a lab that are constantly changing," says marketing manager Adrian Fergus. "What's important is to provide open hooks from the LIMS to allow it to quickly and easily integrate with new robotic equipment and new applications."

Such flexible LIMS are likely to become a necessity for many drug labs because of the ruling by the US Food and Drug Administration that data on drug development must be kept in a format that will be accessible for evaluation no matter how data-storage technology evolves. Although this legislation has been in force since 1997, enforced compliance is only now being phased in, prompting a new wave of compliant systems. For example, Hamilton, a developer of fluid-handling systems in Reno, Nevada, has recently launched a new version of the controlling software for its Microlab robotic instruments.

As well as being sold as a 'ready-to-run' software package, Nautilus is also available as an application service, where labs pay a monthly fee for a hosted LIMS service delivered through the Internet. "It generally appears to be smaller labs that have shown an interest, where they do not have the IT resources in-house to manage a LIMS and are willing to have that system hosted," Fergus says. "What it avoids is the initial upfront cost of the licences and so spreads the cost more evenly and is more manageable."

As with all areas of laboratory automation, the aim of LIMS is to increase productivity and efficiency, says Felder. "The motivation in this industry is not eliminating jobs, it is getting products to market faster. This is one case where automation will create jobs rather than eliminating them," he says.

Tim Chapman is a freelance journalist based in Halifax, UK. 